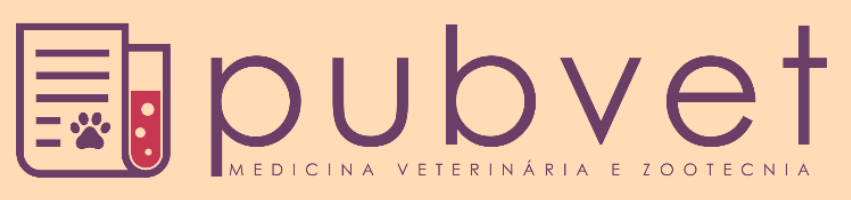

https://doi.org/10.31533/pubvet.v15n11a973.1-6

\title{
Elaboração e análise sensorial de hambúrguer suíno com implementação de lecitina de soja e redução de sódio
}

\author{
Raquel Martins Trindade ${ }^{\oplus}$, Ana Carolina Duque ${ }^{1} \bullet$, Renato Luiz Silveira ${ }^{2} \theta$, Silvio Jorge \\ Machado $^{3} \bullet$, Fabiana Batalha Knackfuss ${ }^{4 *} \bullet$, Rami Fanticelli Baptista ${ }^{4}$ \\ ${ }^{I}$ Aluna do Curso de Medicina Veterinária da Universidade do Grande Rio- UNIGRANRIO, Duque de Caxias, Rio de Janeiro, Brasil. \\ ${ }^{2}$ Professor do Departamento de Morfologia, Instituto Biomédico, UFF, Niterói, Rio de Janeiro, Brasil. \\ ${ }^{3}$ Resonsável Técnico do Laboratório Multidisciplinar da UNIGRANRIO, Duque de Caxias, Rio de Janeiro, Brasil. \\ ${ }^{4}$ Professora do Curso de Medicina Veterinária da Universidade do Grande Rio- UNIGRANRIO, Duque de Caxias, Rio de Janeiro, Brasil. \\ *Autor para correspondência: knackfussfb@unigranrio.edu.br
}

Resumo. O objetivo do presente estudo foi elaborar quatro tratamentos de hambúrguer de pernil suíno e avaliar com a participação de provadores não treinados, os parâmetros de aparência, sabor, textura, cor e aroma através da análise sensorial de aceitação por escala hedônica de nove pontos, além de examinar a intenção de compra e de indicação para cada um dos tratamentos, sendo o tratamento A controle, tratamento B com redução parcial do teor de sódio, tratamento $\mathrm{C}$ com a implementação de lecitina de soja e tratamento D com implementação de lecitina de soja e redução parcial do teor de sódio. Para a formulação base foi realizada uma mistura de coentro, alho, cebola, pimenta branca, ácido ascórbico, adicionada em pernil suíno e água. De acordo com o teste de Friedman, o tratamento D obteve os melhores resultados para o atributo de aparência e o sabor foi um quesito que apresentou diferença estatística significativa $(p<0,05)$ indicando que houve um maior impacto para aceitação dos consumidores, e, além disso, maiores intenções de compra e recomendação ao produto, onde o tratamento $\mathrm{B}$, juntamente com a formulação controle A, obtiveram as melhores médias para o atributo sabor. A utilização da lecitina de soja agregou além de valor ao produto, uma melhor aparência, que agradou o consumidor; porém, o atributo sabor apresentou maior relevância quanto à preferência dos consumidores.

Palavras-chave: Análise sensorial, hambúrguer, lecitina de soja, pernil suíno, redução de sódio

\section{Preparation and sensory analysis of pork hamburger with implementation of soy lecithin and reduction of sodium}

\begin{abstract}
The objective of the present study was to elaborate four pork hamburger treatments and to evaluate the parameters of appearance, taste, texture, color and aroma with the participation of untrained tasters through the sensorial analysis of acceptance by hedonic scale of nine points, in addition to examining the intention to purchase and indication for each treatment, formulation $\mathrm{A}$, control treatment, treatment $\mathrm{B}$ with partial reduction of sodium content, treatment $\mathrm{C}$ with the implementation of soy lecithin and treatment $\mathrm{D}$ with implementation of soy lecithin and partial reduction of the sodium content. For the base formulation was made a mixture of coriander, garlic, onion, white pepper, ascorbic acid, added in pork leg and water. According to the Friedman test, treatment D obtained the best results for the appearance attribute. And the flavor was a question that presented a significant statistical difference $(\mathrm{P}<0,05)$, indicating that there was a greater impact for consumer acceptance, and, in addition, greater intentions of purchase and recommendation to the product, where treatment $\mathrm{B}$, together with the
\end{abstract}


control formulation A, obtained the best means for the flavor attribute. It was concluded that the use of soy lecithin added, besides a value to the product, a better appearance, which pleased the consumer, but the flavor attribute presented greater relevance as the consumers preference.

Keywords: Hamburger, pork leg, sensory analysis, sodium reduction, soy lecithin

\section{Introdução}

A carne suína, além de ter alta palatabilidade, é rica em nutrientes e fornece grande benefício para a saúde humana (Ludtke et al., 2010; Rosenvold \& Andersen, 2003). Sua composição nutricional engloba elevado teor de proteínas com alto valor biológico, ácidos graxos monoinsaturados, além de minerais como ferro e selênio e vitaminas do complexo B (Moura et al., 2015), sendo, inclusive, a principal fonte de tiamina quando comparado a carne bovina e de frango (Magnoni \& Pimentel, 2006).

Levando-se em consideração que o consumo excessivo de sal de cozinha (cloreto de sódio), pode desencadear problemas de saúde como a obesidade e hipertensão arterial, considerados fatores de risco para acidente vascular cerebral, infarto, insuficiência renal e cardíaca (Schönfeldt \& Gibson, 2008), a Organização Mundial de Saúde (OMS) recomenda que deve, ser utilizado 1,2 grama de sal para as necessidades nutricionais diárias. Ainda de acordo com Pinheiro et al. (2013), uma das recomendações é que a relação potássio-sódio seja baixa, sendo que a carne suína possui a razão potássio-sódio em torno de 7, ou seja, altamente favorável ao controle da pressão arterial. Sendo assim, a redução do teor de sódio na alimentação da população para o controle da hipertensão de grande importância (Ministério da Saúde, 2019).

Para a redução parcial do teor de sódio e manutenção da palatabilidade, uma das possibilidades é a utilização do cloreto de potássio, sendo o principal substituto e mais usado. Por esse motivo, e por apresentar propriedades semelhantes ao sal (cloreto de sódio). Essa substituição deve ser parcial, pois o cloreto de potássio pode conceder sabor amargo ao alimento (Carvalho et al., 2015; Paulino et al., 2006), e como benefício, os efeitos que são gerados pelo sódio na pressão arterial serão reduzidos (Vogel et al., 2011), tornando o produto aceitável para os consumidores que procuram por um alimento saudável (Nascimento et al., 2008).

Um outro componente utilizado como aditivo em produtos industrializados é a lecitina de soja, que é um subproduto da soja, considerado um produto funcional, e que possui um grande valor comercial, pois é usado tanto nas indústrias alimentícias como nas farmacêuticas. Entre seus benefícios está o combate à obesidade, auxílio ao controle da pressão arterial e hipercolesterolemia. A lecitina tem, em sua composição, fosfatidilcolina um fosfolipídio muito nutritivo, fundamental para reduzir o teor de colesterol plasmático de lipídios (Ramalho \& Suarez, 2013).

O objetivo do presente estudo foi elaborar quatro tratamentos de hambúrgueres de pernil suíno, contendo cloreto de potássio como substituto parcial do cloreto de sódio e a implementação da lecitina de soja para posterior análise sensorial por meio do método de aceitação por escala hedônica de nove pontos, bem como verificar a intenção e indicação de compra para cada um dos tratamentos.

\section{Material e métodos}

Foram adquiridos seis quilogramas de pernil suíno com osso e pele, congelados e embalados de uma marca comercial, com selo do Serviço de Inspeção Federal (SIF), em um mercado atacadista na cidade de Duque de Caxias/RJ. Em seguida, as embalagens foram conduzidas em um continente isotérmico até a chegada ao laboratório multidisciplinar da Universidade do Grande Rio (UNIGRANRIO), Duque de Caxias.

No laboratório a carne foi armazenada em geladeira durante 72 horas em temperatura de $2^{\circ} \mathrm{C}$, para obter o descongelamento lento. Após o descongelamento, foram retirados gorduras e peles da carne, e cortada em partes menores, para ser direcionadas ao moedor industrial da marca Skymsen ${ }^{\circledR}$. Os pedaços passaram por moagem em disco de dez milímetros, e posteriormente, em disco de cinco milímetros. A carne foi pesada em balança de bancada da marca Toledo ${ }^{\circledR}$ e constavam quatro quilogramas de pernil. Esta carne foi dividia em quatro porções, de um quilograma, para os seguintes 
tratamentos: A, B, C e D. Os ingredientes descritos na Tabela 1 foram selecionados e pesados em balança da marca Gehaka ${ }^{\circledR}$.

Para a formulação base foi realizado uma mistura de coentro $(0,2 \%)$, alho $(1 \%)$, cebola $(1 \%)$, pimenta branca $(0,2 \%)$, ácido ascórbico $(0,2 \%)$ sendo adicionada em carne de pernil suíno $(88,4 \%)$ e água $(3,5 \%)$.

Tabela 1. Quantidade dos ingredientes utilizados no preparo de hambúrguer de pernil suíno demonstrados em porcentagem

\begin{tabular}{lcccc}
\hline Ingredientes & $\mathrm{A}$ & $\mathrm{B}$ & $\mathrm{C}$ & $\mathrm{D}$ \\
\hline Água & $3.5 \%$ & $3.5 \%$ & $3.5 \%$ & $3.5 \%$ \\
Alho em pó & $1 \%$ & $1 \%$ & $1 \%$ & $1 \%$ \\
Ácido ascórbico & $0,2 \%$ & $0,2 \%$ & $0,2 \%$ & $0,2 \%$ \\
Cebola em pó & $1 \%$ & $1 \%$ & $1 \%$ & $1 \%$ \\
Coentro & $0,2 \%$ & $0,2 \%$ & $0,2 \%$ & $0,2 \%$ \\
Cloreto de potássio & - & $0,4 \%$ & $1,5 \%$ & $0,4 \%$ \\
Cloreto de sódio & $1,5 \%$ & $1,1 \%$ & $4 \%$ & $1,1 \%$ \\
Lecitina de soja & - & - & $88,4 \%$ & $4 \%$ \\
Pernil suíno & $88,4 \%$ & $88,4 \%$ & $0,2 \%$ & $88,4 \%$ \\
Pimenta branca & $0,2 \%$ & $0,2 \%$ & - & $0,2 \%$ \\
Toucinho & $4 \%$ & $4 \%$ & - \\
\hline
\end{tabular}

$\mathrm{O}$ tratamento A, foi formulado com todos os ingredientes base, este tratamento foi usado como o controle, pois é o que mais se aproxima das formulações padrão. Neste tratamento, o cloreto de sódio foi utilizado na proporção de $1,5 \%$, em relação ao peso da carne moída que foi de um quilograma. Portanto, o cloreto de sódio utilizado no tratamento A, foi de quinze gramas. Para o tratamento B, além dos ingredientes base, possui cloreto de potássio, este tratamento foi elaborado para avaliar a redução do teor de sódio, utilizando $1,1 \%$ de cloreto de sódio e $0,4 \%$ de cloreto de potássio. $\mathrm{O}$ tratamento $\mathrm{C}$ foi elaborado com os ingredientes base, o cloreto de sódio foi usado em proporção de $1.5 \%$ ao peso da carne moída, e neste tratamento foi feito à implementação de $4 \%$ de lecitina de soja e para o tratamento $\mathrm{D}$ em sua formulação possui todos os ingredientes base, além disso, foi adicionado o cloreto de sódio na proporção de $1,1 \%$, o cloreto de potássio em $0,4 \%$ e também, $4 \%$ de lecitina de soja.

A carne moída foi colocada em um recipiente, onde todos os ingredientes foram adicionados junto a carne, homogeneizando até chegar à consistência firme. Esta etapa foi realizada com os quatros tratamentos.

Foram pesados 60 quilogramas de carne em balança de bancada da marca Toledo ${ }^{\circledR}$, moldados em forma esférica e em seguida foram envoltas em plástico de polietileno, como sendo a embalagem primária. Para moldar os hambúrgueres de carne suína foi utilizado um molde de formato redondo medindo 90 x 15 milímetros e, posteriormente, embalados individualmente em embalagem secundária de plástico de polietileno de baixa densidade e, selados. Cada embalagem recebeu etiqueta de identificação de acordo com seus respectivos tratamentos.

Os hambúrgueres foram submetidos ao congelamento, durante 48 horas, em temperatura máxima de $-30^{\circ} \mathrm{C}$. Dez minutos antes do preparo, os hambúrgueres foram retirados do congelador e colocados na bancada em temperatura ambiente. Após os dez minutos, foram retiradas das embalagens primárias e secundárias, e em seguida, embrulhados com papel alumínio para serem levados ao forno. Em um tabuleiro de aço inoxidável forrado com papel alumínio, foram colocados os hambúrgueres. Foi utilizado um forno industrial da marca Metalmaq ${ }^{\circledR}$, pré-aquecido durante 20 minutos na temperatura de $240^{\circ} \mathrm{C}$.

Os tabuleiros identificados para cada tratamento, foram colocados ao forno durante 10 minutos para cada lado do hambúrguer, na temperatura de $250^{\circ} \mathrm{C}$. Todos os tratamentos foram assados no mesmo momento e no mesmo forno. Após o cozimento, os hambúrgueres foram retirados do forno e armazenados em recipiente de aço inoxidável até chegar à temperatura ambiente para poder ser consumido. 
Para realização da análise sensorial, foram convidados como provadores não treinados, alunos, docentes e funcionários da Universidade do Grande Rio, localizada em Duque de Caxias. As análises sensoriais foram realizadas nos dias nove e dez de maio de 2019, no laboratório multidisciplinar da Universidade do Grande Rio. Seguindo os mesmos cronogramas nos dois dias, participaram no total de 71 provadores.

As orientações foram feitas individualmente, com instruções sobre a análise sensorial. Para cada participante foi entregue e explicado o termo de consentimento livre e esclarecido para que o mesmo tomasse conhecimento sobre todas as etapas a serem feitas durante análise, e assim estando de acordo assinasse o termo. Posteriormente, os provadores foram conduzidos até uma cabine individual de análise sensorial.

Cada provador recebeu quatro fichas referentes aos quatro tratamentos, e um copo contendo $80 \mathrm{Ml}$ de água, com cinco gotas de limão, com objetivo de realizar uma limpeza das papilas gustativas, indicando o mesmo procedimento entre a degustação de cada tratamento. As amostras foram servidas no formato de $1 / 4$ de hambúrguer em pratos plásticos codificados de acordo com cada tratamento (A, B, C, D), e foram distribuídas de forma aleatória.

O teste de aceitação por escala hedônica foi realizado de acordo com Cruz et al. (2013). Os provadores analisaram a partir dos aspectos de aparência, sabor, textura, cor, e aroma de cada amostra para cada tratamento. Para a análise dos atributos foi utilizada a escala hedônica de nove pontos (1 desgostei extremamente, 2 - desgostei moderadamente, 3 - desgostei regularmente, 4 - desgostei ligeiramente, 5 - não gostei, nem desgostei, 6 - gostei ligeiramente, 7 - gostei regularmente; 8 gostei moderadamente e 9 - gostei extremamente).

Outro teste que foi aplicado paralelo ao de aceitação de compra, foi o de intenção de compra, onde cada voluntário respondeu se compraria ou não, e se indicaria ou não o produto pelo qual provou com as seguintes respostas fechadas (sim ou não).

Para a avaliação dos dados obtidos pela análise sensorial, foi utilizado o teste de Friedman, a partir do programa Bioestat 5.0.

\section{Resultados e discussão}

A partir do teste de Friedman verificou-se as comparações das médias do Ranking entre os quatro tratamentos. Os resultados das análises sensoriais avaliaram os atributos de cor, sabor, textura, aparência e aroma, das amostras de hambúrgueres A, B, C e D seguindo as diferentes formulações.

Com relação aparência, foi verificada diferença $(P=0,02)$ entre as formulações, sendo que a maior aceitação foi observada para o tratamento D $(2,78)$, comparativamente A $(2,23)$, B $(2,68)$ e C $(2,3)$. Em relação ao quesito aparência, o tratamento D obteve melhores resultados quando comparado ao A $(\mathrm{P}<0,05)$, sugerindo que a implementação de lecitina de soja, de acordo com (Van Nieuwenhuyzen, 1976), usualmente apresenta coloração vermelha acastanhada, o que atribui uma aparência bastante aceita pelos consumidores.

Para os atributos aroma, textura e cor não foram observadas diferenças estatísticas significativas, tendo sido obtidos valores de $\mathrm{p}$ iguais a $0,24,0,91$ e 0,53 respectivamente, indicando que os provadores não conseguiram discernir estes atributos de forma individual, o que corrobora com os resultados obtidos por (Monteiro et al., 2015), que também não adquiriram resultados significativos, quando substituíram de forma parcial $(50 \%)$ do cloreto de sódio por cloreto de potássio em bife reestruturado de tilápia.

Foi detectada diferença $(\mathrm{P}<0,0037)$ entre os tratamentos com relação ao sabor, sendo observadas as seguintes médias para os tratamentos A, B, C e D, respectivamente: 2,75, 2,66, 2,03, 2,55. Fazendo o desdobramento estatístico entre os tratamentos, ficou evidenciado que neste atributo, houve diferença entre A e C e entre B e C, podendo ser justificado segundo Ruusunen \& Puolanne (2005), pois a redução do teor de sal e retirada da gordura animal, diminui o sabor e a aceitação do consumidor pelo produto. Os tratamentos A e B apresentaram as melhores médias quanto ao sabor. 
$\mathrm{Na}$ avaliação de intenção de compra, foi possível observar maiores percentuais de aceitação do que de rejeição, sendo que as formulações A e B receberam as melhores avaliações. Este resultado coincide com as maiores médias para sabor nestes dois tratamentos, o que pode indicar que o sabor foi o item que mais contribuiu para a aceitação e gerou maiores intenções de compra dos consumidores. Um dos atributos que influência na escolha do consumidor quanto a um determinado produto é o sabor (Carvalho et al., 2017; Passetti et al., 2020; Vital et al., 2018).

Com relação à intenção de compra das formulações, o tratamento A de acordo com os resultados, apontam que $77,5 \%$ comprariam e $22,5 \%$ não comprariam, para o tratamento B $80,3 \%$ comprariam e $19,7 \%$ não comprariam, para o tratamento C $57,7 \%$ comprariam e $42,3 \%$ não comprariam, e para o tratamento D 70,4\% comprariam e $29,6 \%$ não comprariam.

Sobre o questionamento se o consumidor indicaria ou não os tratamentos consumidos, os resultados obtidos, foram que $83,1 \%$ dos consumidores recomendariam o tratamento A e $16,9 \%$ não recomendariam, no tratamento B $80,3 \%$ recomendariam este produto e $19,7 \%$ não recomendariam, no tratamento C 54,9\% recomendariam e 45,1\% não recomendariam, e no tratamento $\mathrm{D} 78,9 \%$ recomendariam e $21,1 \%$ não recomendariam. Sendo possível observar pelos resultados que os tratamentos A e B obtiveram melhor indicação, coincidindo com os resultados de Intenção de Compra e sobre o atributo sabor.

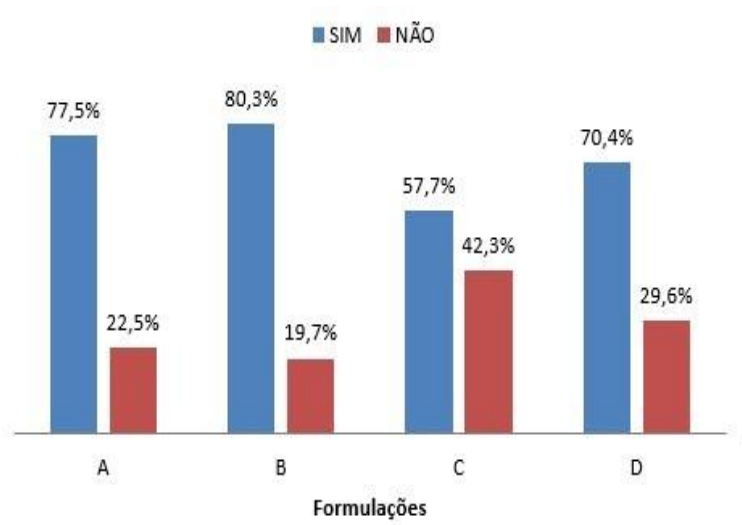

Figura 1. Percentual de respostas com relação à intenção de compra das formulações dos tratamentos A, B, C e D

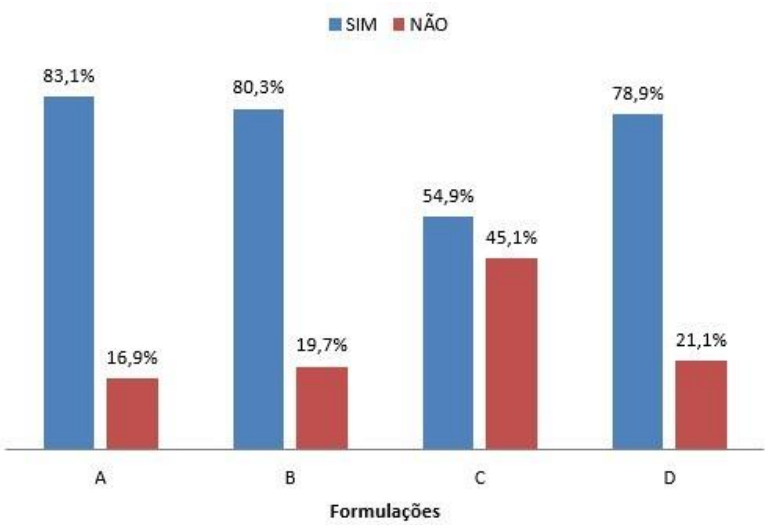

Figura 2. Percentual de respostas com relação à recomendação das formulações A, B, C e D

\section{Conclusão}

O uso de lecitina de soja na formulação do tratamento D tornou o produto mais saudável e com uma aparência mais atrativa ao consumidor. $\mathrm{O}$ atributo sabor apresentou grande importância nos resultados quanto à aceitação, intenção de compra e recomendação ao produto pelos consumidores, sendo que os tratamentos A, sendo o controle, e o tratamento B com redução do teor de sódio obtiveram os melhores resultados. A elaboração de hambúrguer de pernil suíno é uma das alternativas para se aumentar o consumo de carne suína no Brasil.

\section{Referências bibliográficas}

BRASIL. Ministério da saúde. Hipertensão é diagnosticada em $24,7 \%$ da população, segundo a pesquisa Vigitel. Maio. 2019.

Carvalho, C B, Vital, A. C. P., Kempinski, E. M. B. C., Madrona, G. S., Reche, P. M., Guerrero, A., Ornaghi, M. G., \& Prado, I. N. (2017). Quality and sensoria evaluation of beef hamburger made with herbs, spices, and reduced sodium content. Journal of Culinary Sciences \& Technology, 1, 114. https://doi.org/10.1080/15428052.2017.1363108.

Carvalho, Camila Barbosa, Madrona, G. S., Cestari, L. A., Guerrero, A., Souza, N. E., \& Prado, I. N. (2015). Sensory profile of beef burger with reduced sodium content. Acta Scientiarum. Technology, 37(2), 301-305. https://doi.org/10.4025/actascitechnol.v37i2.25224.

Cruz, A. G., Cavalcanti, R. N., Guerreiro, L. M. R., Sant'Ana, A. S., Nogueira, L. C., Oliveira, C. A. F., Deliza, R., Cunha, R. L., Faria, J. A. F., \& Bolini, H. M. A. (2013). Developing a prebiotic 
yogurt: Rheological, physico-chemical and microbiological aspects and adequacy of survival analysis methodology. Journal of Food EEngineering, 114(3), 323-330. https://doi.org/10.1016/j.jfoodeng.2012.08.018.

Ludtke, C. B., Silveira, E. T. F., Bertoloni, W., Andrade, J. C., Buzelli, M. L. T., Bressa, L. R., \& Soares, G. J. D. (2010). Bem-estar e qualidade de carne de suínos submetidos a diferentes técnicas de manejo pré-abate. Revista Brasileira de Saúde e Produção Animal, 11(1), 231-241.

Magnoni, D., \& Pimentel, I. (2006). A importância da carne suína na nutrição humana. In UNIFEST.

Monteiro, M. L. G., Mársico, E. T., Canto, A. C. V. C. S., Costa-Lima, B. R. C., Lázaro, C. A., Cruz, A. G., \& Conte Júnior, C. A. (2015). Partial sodium replacement in tilapia steak without loss of acceptability. Food Science and Technology International, 21(4), 295-305. https://doi.org/10.1177/1082013214535229.

Moura, J. W. F., Medeiros, F. M., Alves, M. G. M., \& Batista, A. S. M. (2015). Fatores influenciadores na qualidade da carne suína. Revista Científica de Produção Animal, 17(1), 18-29.

Nascimento, R. do, Campagnol, P. C. B., Monteiro, E. S., \& Pollonio, M. A. R. (2008). Substituição de cloreto de sódio por cloreto de potássio: influência sobre as características físico-químicas e sensoriais de salsichas. Alimentos e Nutrição Araraquara, 18(3), 297-302.

Passetti, R. A. C., Macedo, F. A. F., Santos, G. R. A., Bonin, E., Vital, A. C., Ramos, T. R., Passetti, L. C. G., Ornaghi, M. G., Costa, I. C. A., \& Prado, I. N. (2020). Sensorial, color, lipid oxidation, and visual acceptability of dry-aged beef from young bulls with different fat thickness. Animal Science Journal, 91(e-13498), 1-10. https://doi.org/10.1111/asj.13498.

Paulino, F. O., Silva, T. J. P., Franco, R. M., Freitas, M. Q., \& Fernandes, M. L. (2006). Redução parcial dos teores de gordura e sal em embutido cárneo suíno com utilização de goma carragena e cloreto de potássio. Revista Brasileira de Ciência Veterinária, 13(2), 121-124.

Pinheiro, R. E. E., Cardoso, E. C., Júnior, M. H. K., Muratori, M. C. S., Lopes, J. B., Farias, L. A., \& Teixeira, M. P. F. (2013). Qualidade da carne de suínos mestiços comerciais e sem raça definida criados em regime intensivo. Revista Brasileira de Saúde e Produção Animal, 14, 149-160.

Ramalho, H. F., \& Suarez, P. A. Z. (2013). A Química dos óleos e gorduras e seus processos de extração e refino. Revista Virtual de Química, 5(1), 2-15. https://doi.org/10.5935/19846835.20130002.

Rosenvold, K., \& Andersen, H. J. (2003). Factors of significance for pork quality-a review. Meat Science, 64(3), 219-237. https://doi.org/http://dx.doi.org/10.1016/S0309-1740(02)00186-9

Ruusunen, M., \& Puolanne, E. (2005). Reducing sodium intake from meat products. Meat Science, 70(3), 531-541.

Schönfeldt, H. C., \& Gibson, N. (2008). Changes in the nutrient quality of meat in an obesity context. Meat Science, 80(1), 20-27. https://doi.org/http://dx.doi.org/10.1016/j.meatsci.2008.05.025

Van Nieuwenhuyzen, W. (1976). Lecithin production and properties. Journal of the American Oil Chemists'Society, 53(6Part2), 425-427. https://doi.org/10.1007/BF02605737.

Vital, A. C. P. C. P., Guerrero, A., Ornaghi, M. G. G., Kempinski, E. M. B. C., Sary, C., Monteschio, J. O. O., Matumoto-Pintro, P. T. T., Ribeiro, R. P. P., \& Prado, I. N. N. (2018). Quality and sensory acceptability of fish (Oreochronis niloticus) with alginate-based coating containing essential oils. Journal of Food Science and Technology, 1(12), 1-11. https://doi.org/10.1007/s13197-018-3429-y.

Vogel, C. C., Pazuch, C. M., Sarmento, C., Back, L., \& Secco, T. H. (2011). Desenvolvimento de salsicha com teor de sódio reduzido (sal light). Revista Ciências Exatas e Naturais, 13(3), 305-316.

Histórico do artigo:

Recebido: 18 de julho de 2021

Aprovado: 20 de agosto de 2021
Licenciamento: Este artigo é publicado na modalidade Acesso Aberto sob a licença Creative Commons Atribuição 4.0 (CC-BY 4.0), a qual permite uso irrestrito, distribuição, reprodução em qualquer meio, desde que $\mathrm{o}$ autor $\mathrm{e}$ a fonte sejam devidamente creditados. 